\title{
Conversión acelerada de retama negra (Cytisus scoparius) en un biofertilizante de calidad mediante vermicompostaje
}

Recibido: 9 decembro 2014 / Aceptado: 11 febreiro 2015

(C) IBADER- Universidade de Santiago de Compostela 2014

\begin{abstract}
Resumen Cytisus scoparius es una planta arbustiva ampliamente distribuida por todo el mundo y considerada como una amenaza para otras especies en algunos países. Las plantas del género Cytisus crecen sobre todo en áreas perturbadas y su presencia favorece, a menudo, la propagación rápida del fuego. El objetivo de este estudio fue evaluar el proceso de vermicompostaje de estos arbustos para producir un fertilizante orgánico de alta calidad y libre de polifenoles. El vermicompostaje redujo sustancialmente la biomasa de $C$. scoparius (en un $84 \%$ ), fundamentalmente como resultado de la pérdida de sólidos volátiles, en tan sólo 6 semanas. Se obtuvo un vermicompost de calidad, que es un material similar a la turba, rico en nutrientes y microbiológicamente activo y estable, y sin la fitotoxicidad asociada a los polifenoles. Simultáneamente, la población inicial de lombrices se incrementó muy considerablemente, en un $350 \%$. El procedimiento descrito es eficaz, simple y económico, y puede escalarse fácilmente para su aplicación industrial.
\end{abstract}

Palabras clave Residuo vegetal, compuestos polifenólicos, vermirreactor, Eisenia andrei, vermicompost.

Abstract Wild shrubs belonging to Cytisus scoparius are widely distributed throughout the world, and they are considered a threat to other species in some countries. Cytisus spp. mainly grow in disturbed areas and their

Hugo Martínez-Cordeiro · Jorge Domínguez

Departamento de Ecoloxía e Bioloxía Animal, Universidade de Vigo, E-36310 Vigo.

Tel: 986814099 Fax: 986812556

E-mail: jdguez@uvigo.es (Jorge Domínguez)

Marta Pájaro Varela · Carmen García-Jares · Marta Lores Departamento de Química Analítica, Nutrición e Bromatoloxía, Universidade de Santiago de Compostela, E-15782, Santiago de Compostela. presence often favours the rapid spread of fire. Theaim of this study was to evaluate whether such shrubs could be processed by vermicomposting on an industrial scale to yield high-quality organic and polyphenol-free fertiliser. Vermicomposting reduced thebiomass of $C$. scoparius substantially (by $84 \%$ ), mainly as a result of the loss of volatile solids. After 6 weeks, the process yielded anutrientrich, microbiologically active and stabilised peat-like material without the polyphenol-associated phytotoxicity. Simultaneously, the initial population of earthworms increases remarkably, up to $350 \%$. The procedure described is effective, simple and economical, and could easily be scaled up for industrial application.

Key words Vegetable wastes, polyphenolic compounds, vermirreactor, Eisenia andrei, vermicompost.

\section{Introducción}

España tiene la tercera mayor extensión de superficie arbolada de la Unión Europea con 18,4 millones de hectáreas equivalentes al $36,3 \%$ del territorio nacional. La superficie desarbolada de España es de 9,3 millones de hectáreas de las 20,3 millones de hectáreas de la Unión Europea, es decir, casi la mitad de los matorrales y pastizales europeos están en nuestro país, cubriendo el $18,5 \%$ de la superficie nacional (MAGRAMA, 2014). En Galicia, la superficie de matorral ocupa unas 800000 hectáreas. Un problema importante de los últimos años es la pérdida de grandes masas de bosque debido a los incendios forestales; dejando numerosas zonas devastadas que permiten la expansión de especies vegetales menos selectivas como los matorrales, que son a su vez causa del origen y propagación de los incendios. Por otra parte, el éxodo rural deja en desuso grandes áreas de terreno agrícola que son invadidas por matorrales. Además, las campañas de explotación forestal mal planificadas suponen la deforestación de zonas amplias de bosque y la invasión posterior de estas especies oportunistas (Núñez-Regueira et al. 2004). 
Una de las especies que se propagan con gran facilidad en las condiciones anteriormente comentadas es la retama negra (Cytisus scoparius), planta arbustiva perteneciente a la familia Fabaceae. Esta planta es de origen europeo, y se extiende entre España y el sur de Suecia y entre Irlanda y Ucrania. En Francia se considera una planta invasora para la agricultura y los ecosistemas naturales, llegando a suponer una amenaza por su capacidad para colonizar pastos abandonados (Prévosto et al. 2004). La retama negra ha sido introducida en numerosos países como Canadá, Chile, Australia, India, Irán, Nueva Zelanda, Sudáfrica y Estados Unidos (Peterson \& Prasad 1998) reduciendo la biodiversidad de los ecosistemas naturales y convirtiéndose en un serio problema por la pérdida de plantaciones forestales; ya que puede inhibir la germinación de las semillas y ralentizar el crecimiento de los árboles jóvenes (Potter et al. 2009).

La retama negra se caracteriza por sus brillantes flores amarillas, que aparecen solas o juntas en las axilas de las hojas; y por sus frutos, vainas con pelos marginales que contienen de 5 a 9 semillas (Peterson \& Prasad 1998). Esta planta forma masas densas en zonas abiertas, perturbadas por incendios o talas de árboles, aunque crece también de forma natural en prados, claros de bosque y matorrales. Se trata de una leguminosa con una gran capacidad para fijar nitrógeno (Downey \& Smith 2000; Prasad 2003), con un alto contenido en fósforo, potasio y calcio (Gatica-Trabanini \& Rodríguez 1997) y con un considerable contenido polifenólico (Barros et al. 2012). Este elevado contenido en nutrientes hace de la retama negra un recurso potencial para ser utilizado como fertilizante orgánico en los suelos agrícolas. Sin embargo, la aplicación directa de este material "crudo" puede dañar los cultivos debido a la liberación de polifenoles que en cantidades excesivas son fitotóxicos (Inderjit 1996).

El objetivo de este trabajo fue evaluar el potencial del vermicompostaje para convertir de forma acelerada la retama negra en un biofertilizante para agricultura.

\section{Materiales y métodos}

Retama Negra (Cytisus scoparius) y lombrices de tierra

La retama utilizada se recolectó manualmente en Galicia durante la primavera del año 2014 con la planta en plena floración. Las flores y las hojas no se trocearon; las ramas jóvenes se trocearon con una máquina trituradora (tamaño de partícula entre 3 y $6 \mathrm{~cm}$ ). Se utilizaron lombrices de tierra de la especie Eisenia andrei Bouché (1972), pertenecientes a la categoría ecológica de las epigeas; estas lombrices viven en el horizonte orgánico del suelo y son de pequeño tamaño, pigmentadas, con una alta tasa reproductiva, ciclo de vida corto, alta tasa de consumo y una elevada tasa de asimilación de la materia orgánica en descomposición.

\section{Funcionamiento del vermirreactor}

El procesamiento de la retama negra tuvo lugar en un vermirreactor con una superficie de $6 \mathrm{~m}^{2}(1,5 \times 4 \mathrm{~m})$ que contenía una cama de vermicompost maduro de $12 \mathrm{~cm}$ de espesor con una población total de lombrices de $280 \pm 9$ individuos $\mathrm{m}^{-2}$, incluyendo $111 \pm 10$ lombrices maduras $\mathrm{m}^{-2}$, $169 \pm 7$ juveniles $\mathrm{m}^{-2}$ y $120 \pm 3$ capullos $\mathrm{m}^{-2}$, con una biomasa de 79,1 $\pm 5,2 \mathrm{~g} \mathrm{~m}^{-2}$ (Figura 1). Encima de la cama se dispuso una red plástica de $5 \mathrm{~mm}$ de luz de malla, que permite el paso de las lombrices a la capa de retama extendida homogéneamente sobre ella sin afectar de este modo al procesado del residuo y para facilitar la toma de muestras durante el proceso de vermicompostaje. La capa de retama, de $12 \mathrm{~cm}$ de espesor, se cubrió con una malla de sombra que reduce las pérdidas de agua, manteniéndola con una humedad óptima para las lombrices.

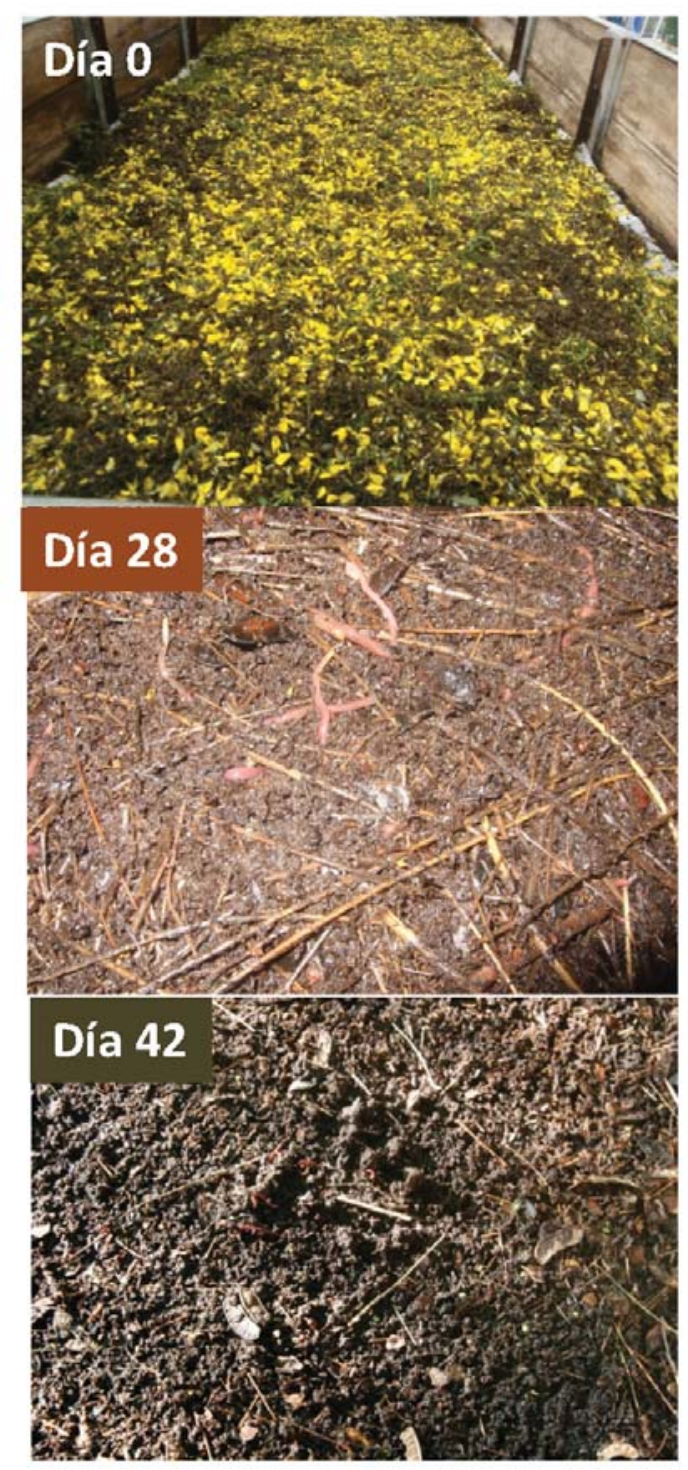

Figura 1.- Reactor de vermicompostaje de retama negra. Las imágenes muestran la evolución de la retama durante el proceso 


\section{Población de lombrices}

Para el seguimiento de la población de lombrices, cada 14 días se recogieron 5 muestras de retama con un espesor de $6 \mathrm{~cm}$ por encima de la malla donde estaba el residuo y $6 \mathrm{~cm}$ por debajo en el mismo sitio correspondiente a la zona de la cama. Las muestras fueron siempre de $6 \mathrm{~cm}$ de espesor a partir de la malla y se recogieron aleatoriamente por todo el vermirreactor utilizando un cilindro de $7,5 \mathrm{~cm}$ de diámetro y $12 \mathrm{~cm}$ de altura. La densidad de las lombrices (Eisenia andrei) se determinó tanto sobre las lombrices totales como separadamente sobre las lombrices maduras, las inmaduras y los capullos.

\section{Toma de muestras}

Semanalmente y de forma aleatoria se recogieron 5 muestras de $10 \mathrm{~g}$ cada una del contenido del vermireactor (retama vermicompostada) para la caracterización química y biológica y el análisis de polifenoles. Las muestras se almacenaron en bolsas de plástico a $-20{ }^{\circ} \mathrm{C}$ hasta la realización de los análisis.

\section{Análisis químicos y biológicos}

La humedad se determinó mediante el método gravimétrico por pérdida de peso a $105^{\circ} \mathrm{C}$ durante 24 horas. El pH y la conductividad eléctrica se midieron en disoluciones acuosas en proporción 1:10 (peso fresco: volumen). El contenido de materia orgánica se determinó por pérdida de peso tras la calcinación de la muestra seca a $550 \pm 50^{\circ} \mathrm{C}$ durante 4 horas en un horno-mufla. La concentración de $\mathrm{C}$ y de $\mathrm{N}$ total se determinaron en muestra seca con un analizador elemental CHNS-O EA1108; y las concentraciones totales de P, K, Ca, Mg, S, Fe, $\mathrm{Mn}, \mathrm{B}$, Mo en muestras secas, sometidas previamente a digestión ácida en microondas, mediante Espectrometría de Emisión Óptica con Plasma Acoplado Inductivamente (ICP-OES). La actividad microbiana se determinó midiendo el consumo de oxígeno con el Sistema de Control ${ }^{\circledR}$ OxiTop (WTW, Weilheim, RFA) de acuerdo con la norma DIN ISO 16072. El contenido total de celulosa, hemicelulosa y lignina se determinó por el sistema FibreBag ${ }^{\circledR}$ (Gerhardt, Königswinter, Alemania) de acuerdo con el método de Goering y Van Soest (1970). Para la determinación de la fibra ácido detergente (FAD) se realizó una digestión de 1 hora en una solución ácida a base de ácido sulfúrico $0,5 \mathrm{M}$ en ebullición. Finalmente, para la determinación de la lignina ácido detergente (LAD) se sigue el mismo procedimiento que para la determinación de la FAD seguido de una digestión de tres horas en ácido sulfúrico al $72 \%$.

\section{Extracción de polifenoles}

Los polifenoles se extrajeron mediante la técnica de extracción con disolventes presurizados (PSE) con un equipo Dionex (ASE 150). La muestra de retama y el dispersante (arena) (1:2) se molieron en un mortero de vidrio para favorecer la ruptura de los tejidos y la posterior extracción de los compuestos fenólicos. Esta mezcla se depositó en la celda de extracción sobre $1 \mathrm{~g}$ de arena y se cubrió con arena. El disolvente de extracción fue agua: metanol (1:1). La celda se introduce en el sistema PSE y se extrae con un $50 \%$ de disolvente orgánico (metanol) en agua. Los extractos obtenidos se enrasaron a $25 \mathrm{~mL}$ con metanol, se filtraron con filtros de $0,22 \mu \mathrm{m}$ y se almacenaron a $-20^{\circ} \mathrm{C}$.

Determinación del índice de polifenoles totales (IPT)

El contenido polifenólico total se determinó con el método de Folin-Ciocalteu, mezclando $5 \mathrm{mLdel}$ extracto (diluido en agua milli-Q, 1:200, 1:100 o 1:50 según contenido fenólico) con $100 \mu \mathrm{l}$ del reactivo Folin \& Ciocalteu y $1 \mathrm{ml}$ de una disolución de carbonato sódico $\left(20 \% \mathrm{Na}_{2} \mathrm{CO}_{3}\right.$ en $\mathrm{H}_{2} \mathrm{O}$ Milli-Q). Se agitó en vortex, se dejó reposar $30 \mathrm{~min}$ a temperatura ambiente en oscuridad y se midió la absorbancia a $760 \mathrm{~nm}$ (Shimazdu, UVmini-1240, Tokio, Japón). La concentración total de polifenoles se calculó utilizando ácido gálico como patrón y se expresó para peso seco de muestra (mg GAEg-1p.s.).

\section{Identificación y cuantificación de los polifenoles}

Los extractos se analizaron por LC-MS/MS en un sistema Thermo Scientific (San Jose, CA, USA) equipado con un espectrómetro de masas de triple cuadrupolo Quantum Access con fuente caliente de ionización por electrospray (HESI). Se utilizó una columna Thermo Scientific Hypersil Gold aQ (1.9 m, $100 \mathrm{~mm} \times 2.1 \mathrm{~mm})$ en un Sistema de HPLC con autoinyector Accela. El volumen de inyección fue de $10 \mathrm{~L}$. Los constituyentes de la fase móvil fueron (A) $0,1 \%$ ácido fórmico en agua y (B) $0,1 \%$ ácido fórmico en metanol. El gradiente programado empezó en $5 \%$ B, cambió a $20 \%$ B a los $2 \mathrm{~min}$, a $70 \%$ B a los $15 \mathrm{~min}$ y finalmente a $100 \%$ B a los $16 \mathrm{~min}$, donde se mantiene 3 min más. El tiempo de análisis fue de $19 \mathrm{~min}$ a un flujo de $300 \mathrm{~L} \mathrm{~min}-1$ y a una temperatura constante de $30^{\circ} \mathrm{C}$.

Los parámetros del MS y de la fuente HESI fueron: $t^{a}$ de vaporización de la fuente $350^{\circ} \mathrm{C}, t^{a}$ del capilar $320^{\circ} \mathrm{C}$, voltaje de electrospray $3000 \mathrm{~V}$ en polaridad positiva y 2800 $\checkmark$ en negativa y la corriente de descarga de la fuente de iones fue 4.0 A para ambas polaridades. De los 18 compuestos detectados, se confirmó la identidad de 7: ácido protocatéquico $(\mathrm{m} / \mathrm{z}$ ion padre $=152 ; \mathrm{m} / \mathrm{z}$ iones producto $=108,109 ; \mathrm{ESI}) ;$ 3,4-dihidroxibenzaldehido $(\mathrm{m} / \mathrm{z}$ ion padre $=137 ; \mathrm{m} / \mathrm{z}$ iones producto $=91,92,136 ;$ ESI- $)$; ácido cafeico $(\mathrm{m} / \mathrm{z}$ ion padre $=179 ; \mathrm{m} / \mathrm{z}$ iones producto $=$ 134,135; ESI-); quercetina-3-glucurónido $(\mathrm{m} / \mathrm{z}$ ion padre $=$ 479; $\mathrm{m} / \mathrm{z}$ iones producto $=303,461 ; \mathrm{ESI})$; rutina, $(\mathrm{m} / \mathrm{z}$ ion padre $=609 ; \mathrm{m} / \mathrm{z}$ iones producto $=179,271,300 ; \mathrm{ESI}-)$; apigenina $(\mathrm{m} / \mathrm{z}$ ion padre $=269 ; \mathrm{m} / \mathrm{z}$ iones producto $=117$, $149,151,225$; ESI-) y crisina $(\mathrm{m} / \mathrm{z}$ ion padre $=253 ; \mathrm{m} / \mathrm{z}$ iones producto $=209,145,143,63$; ESI-). 


\section{Análisis estadístico}

Los datos se analizaron mediante análisis de varianza de medidas repetidas (rANOVA) con el tiempo ( 0 a 42 días) como factor intersujetos. Todas las variables medidas cumplieron los criterios de normalidad, homocedasticidad y esfericidad; y las diferencias significativas se determinaron con el test HSD de Tukey utilizando el paquete estadístico SPSS $v 19$.

\section{Resultados y Discusión}

\section{Evolución de la población de lombrices}

La densidad de población inicial de lombrices (día 0) fue de $280 \pm 9$ individuos $\mathrm{m}^{-2}$, incluyendo $111 \pm 10$ lombrices maduras $\mathrm{m}^{-2}, 169 \pm 7$ juveniles $\mathrm{m}^{-2}$ y $120 \pm 3$ capullos $\mathrm{m}^{-2}$, con una biomasa de $79,1 \pm 5,2 \mathrm{~g} \mathrm{~m}^{-2}$ (Figura 2). El número total de lombrices y su biomasa, así como el número de lombrices maduras, juveniles y capullos aumentó de forma muy significativa durante el proceso de vermicompostaje hasta alcanzar un valor máximo de $1265 \pm 20$ individuos $\mathrm{m}^{-2}$ y una biomasa de $400,6 \pm 1,9 \mathrm{~g} \mathrm{~m}^{-2}$ el día 42 (Figura 2). Las lombrices de tierra pertenecientes a la especie $E$. andrei presentan una alta capacidad de reproducción, y su densidad de población aumentó más de cuatro veces en el proceso. Esto se debe al alimento disponible en la retama y se ve reflejado en el consumo de materia prima, produciendo una elevada y rápida degradación de la misma. La población inicial de lombrices de tierra en el vermirreactor fue baja, y aunque aumentó de forma muy considerable, como consecuencia del aporte de alimento en forma de retama, queda todavía lejos de su capacidad máxima. Como E. andrei vive en ambientes donde el mismo material actúa simultaneamente como sustrato y como alimento, la disponibilidad de material mejora las condiciones para el crecimiento y la reproducción de las lombrices, y conduce a la presencia de un número muy alto de individuos. Cuando la disponibilidad de materia orgánica es elevada, la densidad de lombrices epigeas puede llegar a ser muy alta, de hasta 8000 individuos $\mathrm{m}^{-2}$ en estiercol de vaca y 14600 individuos $\mathrm{m}^{-2}$ en estiercol de cerdo (Monroy et al., 2006).

Como consecuencia de la multiplicación de la población de lombrices, en el proceso de vermicompostaje de la retama, además de vermihumus, también se obtiene una alta biomasa de lombrices. Esta importante producción de proteina animal puede tener diversas aplicaciones industriales, entre las que destacan la obtención de ejemplares vivos para cebo de pesca y de harina para alimentación animal. Las proteínas de las lombrices contienen todos los aminoácidos esenciales, incluso en concentraciones superiores a las del pescado o la carne de vacuno (Edwards \& Bohlen 1996).

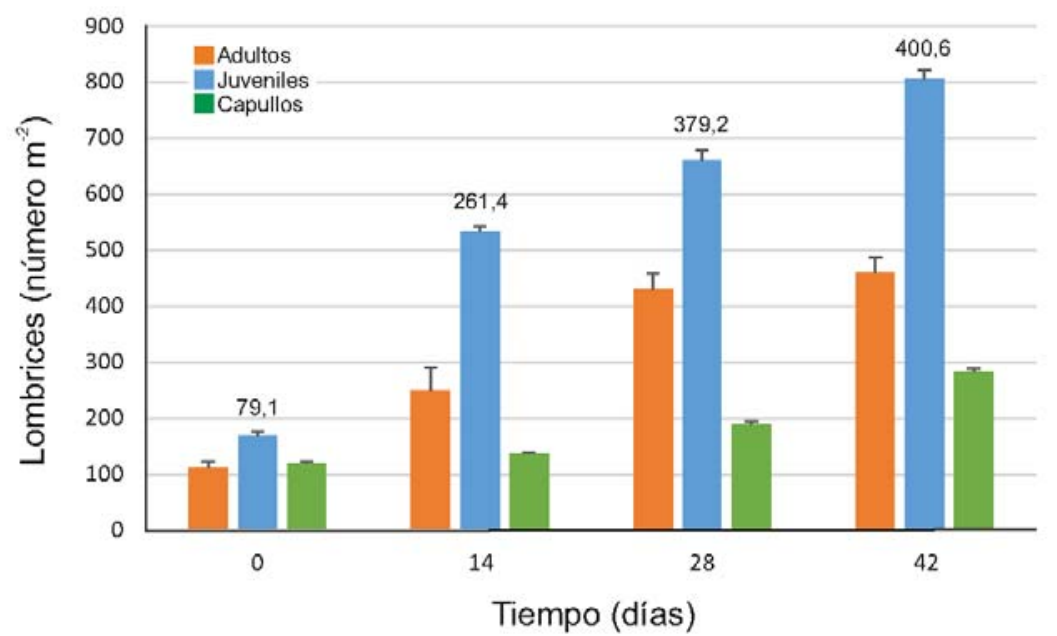

Figura 2.- Densidad de lombrices de tierra (número de adultos, juveniles y capullos por metro cuadrado) y biomasa total de lombrices (gramos $\mathrm{m}^{-2}$ peso fresco, números encima de las barras) durante el vermicompostaje de retama negra. Los valores son medias \pm error estándar $(n=5)$
Aunque los microorganismos son los agentes principales de la descomposición de la materia orgánica, las lombrices de tierran influyen en las tasas de descomposición directamente mediante sus actividades de alimentación y fragmentación, e indirectamente a traves de sus interacciones con los microorganismos (Domínguez 2004; Domínguez et al. 2010). Como consecuencia, las tasas de descomposición están relacionadas directamente con la densidad de población de lombrices (Aira et al. 2002; Aira et al. 2008).
Evolución de las propiedades químicas y biológicas de la retama negra durante el vermicompostaje

Después de los 42 días de proceso de vermicompostaje se determinó el balance de masa total, y la masa inicial de retama negra (120 kg peso fresco; $18,26 \mathrm{~kg}$ peso seco) disminuyó muy significativamente $(83,7 \%)$ como resultado de la pérdida de sólidos volátiles, hasta alcanzar un masa final de $19,6 \mathrm{~kg}$ p.f. (3,77 kg p.s.). Una vez tamizado el producto final se obtuvieron $16 \mathrm{~kg}$ de vermicompost y $3,6 \mathrm{~kg}$ 
de rechazo formado por las fracciones más recalcitrantes de la retama (Figura 1).

El pH de la retama fresca fue de 7,43 0,09 y disminuyó gradualmente hasta el día 42 en el que se estabilizó con un valor de 6,6 0,02 (Tabla 1). La conductividad eléctrica de la retama fresca fue de $0,46 \mathrm{mS} \mathrm{cm}^{-2}$ y también disminuyó de forma gradual durante el vermicompostaje hasta alcanzar un valor de $0,20 \mathrm{mS} \mathrm{cm}^{-2}$ a los 42 días (Tabla 1). El contenido de materia orgánica de la retama fresca fue de $95,36 \pm 0,46 \%$ y disminuyó gradualmente hasta el día 42 en el que alcanzó un valor de 79,52 \pm 0,51\% (Tabla 1).

\begin{tabular}{|c|c|c|c|c|}
\hline & \multirow{2}{*}{\multicolumn{2}{|c|}{ RETAMA NEGRA }} & \multirow{2}{*}{\multicolumn{2}{|c|}{ VERMICOMPOST }} \\
\hline & & & & \\
\hline & DÍA 0 & DÍA 14 & DÍA 28 & DÍA 42 \\
\hline Humedad & $84,78 \pm 0,60^{2}$ & $83,10 \pm 2,52^{2}$ & $80,27 \pm 0,55^{b}$ & $81,58 \pm 1,01^{\text {ab }}$ \\
\hline $\mathrm{pH}$ & $7,43 \pm 0,09^{a}$ & $7,25 \pm 0,04^{b}$ & $6,99 \pm 0,04^{c}$ & $6,6 \pm 0,02^{d}$ \\
\hline ConductividadEléctrica $\left(\mathrm{mS} \mathrm{cm}^{-2}\right)$ & $0,46 \pm 0,00^{a}$ & $0,40 \pm 0,00^{b}$ & $0,32 \pm 0,00^{c}$ & $0,20 \pm 0,00^{d}$ \\
\hline Materia Orgánica (\%) & $95,36 \pm 0,46^{3}$ & $88,73 \pm 1,06^{b}$ & $86,42 \pm 0,68^{c}$ & $79,52 \pm 0,51^{d}$ \\
\hline Carbono Total ( $\mathrm{g} \mathrm{kg}^{-1}$ peso seco) & $489,40 \pm 2,96^{3}$ & $485,80 \pm 1,55^{b}$ & $476,88 \pm 0,68^{\epsilon}$ & $471,44 \pm 0,45^{d}$ \\
\hline Nitrógeno Total ( $\mathrm{g} \mathrm{kg}^{-1}$ peso seco) & $42,40 \pm 0,52^{2}$ & $42,12 \pm 0,24^{a}$ & $38,74 \pm 0,40^{b}$ & $36,42 \pm 0,22^{c}$ \\
\hline Relación $\mathrm{C} / \mathrm{N}$ & $11,54 \pm 0,17^{a}$ & $11,53 \pm 0,06^{a}$ & $12,31 \pm 0,11^{b}$ & $12,94 \pm 0,08^{c}$ \\
\hline Fósforo Total ( $\mathrm{g} \mathrm{kg}^{-1}$ peso seco) & $2,93 \pm 0,32^{a}$ & $3,02 \pm 0,06^{a}$ & $3,19 \pm 0,07^{b}$ & $3,07 \pm 0,03^{\mathrm{ab}}$ \\
\hline Potasio Total ( $\mathrm{g} \mathrm{kg}^{-1}$ peso seco) & $10,33 \pm 1,19^{a}$ & $9,01 \pm 0,30^{a}$ & $7,32 \pm 0,16^{b}$ & $5,99 \pm 0,14^{c}$ \\
\hline Calcio Total ( $\mathrm{g} \mathrm{kg}^{-1}$ peso seco) & $3,91 \pm 0,42^{a}$ & $5,48 \pm 0,36^{b}$ & $7,19 \pm 0,06^{c}$ & $8,99 \pm 0,18^{d}$ \\
\hline Magnesio Total ( $\mathrm{g} \mathrm{kg}^{-1}$ peso seco) & $2,62 \pm 0,28^{a}$ & $2,88 \pm 0,05^{b}$ & $3,05 \pm 0,03^{c}$ & $2,90 \pm 0,05^{b}$ \\
\hline Azufre Total ( $\mathrm{g} \mathrm{kg}^{-1}$ peso seco) & $2,38 \pm 0,27^{a}$ & $2,88 \pm 0,07^{b}$ & $3,12 \pm 0,03^{c}$ & $3,20 \pm 0,06^{d}$ \\
\hline Hierro Total ( $\mathrm{g} \mathrm{kg}^{-1}$ peso seco) & $0,53 \pm 0,07^{a}$ & $0,79 \pm 0,03^{b}$ & $0,97 \pm 0,02^{c}$ & $1,89 \pm 0,02^{d}$ \\
\hline Manganeso Total ( $\mathrm{g} \mathrm{kg}^{-1}$ peso seco) & $0,35 \pm 0,03^{2}$ & $0,46 \pm 0,02^{b}$ & $0,59 \pm 0,00^{c}$ & $0,64 \pm 0,01^{d}$ \\
\hline Boro Total ( $\mathrm{mg} \mathrm{kg}^{-1}$ peso seco) & $34,2 \pm 3,56^{3}$ & $39,8 \pm 1,53^{\text {ab }}$ & $42,6 \pm 1,60^{b}$ & $40,31 \pm 0,67^{b}$ \\
\hline Molibdeno Total ( $\mathrm{mg} \mathrm{kg}^{-1}$ peso seco) & $1,98 \pm 0,16^{a}$ & $3,69 \pm 0,18^{b}$ & $5,67 \pm 0,16^{c}$ & $7,65 \pm 0,26^{d}$ \\
\hline Respiración Basal $\left(\mathrm{mg} \mathrm{O}_{2} \mathrm{~kg} \mathrm{OM}^{-1} \mathrm{~h}^{-1}\right)$ & $779,89 \pm 9,43^{a}$ & $670,89 \pm 8,36^{b}$ & $522,81 \pm 9,91^{\mathrm{C}}$ & $323,59 \pm 4,14^{d}$ \\
\hline Lignina $\left(\mathrm{g} \mathrm{kg}^{-1}\right.$ peso seco) & $206,04 \pm 4,72^{2}$ & $210,45 \pm 5^{2}$ & $241,94 \pm 5,15^{b}$ & $410,06 \pm 5,86^{c}$ \\
\hline Celulosa ( $\mathrm{g} \mathrm{kg}^{-1}$ peso seco) & $294,88 \pm 13,89^{2}$ & $296,7 \pm 10,2^{a}$ & $292,66 \pm 7,49^{a}$ & $201,54 \pm 5,44^{b}$ \\
\hline Hemicelulosa ( $\mathrm{g} \mathrm{kg}^{-1}$ peso seco) & $258,6 \pm 0,4^{2}$ & $252,4 \pm 6,2^{2}$ & $257,24 \pm 9,23^{a}$ & $84,38 \pm 1,58^{b}$ \\
\hline
\end{tabular}

Tabla 1.- Cambios en las características físicas, químicas y biológicas dela retama negra (Cytisus scoparius) durante el proceso devermicompostaje. Los valores se corresponden con la media \pm el error estándar $(n=5)$. Letras diferentes indican diferencias significativas entre los valores(test post hoc Tukey HSD

El contenido de carbono total de la retama fresca fue de $489,40 \pm 2,96 \mathrm{~g} \mathrm{~kg}^{-1}$ p.s. y disminuyó de forma gradual y significativa durante el vermicompostaje hasta alcanzar un valor de $471,44 \pm 0,45 \mathrm{~g} \mathrm{~kg}^{-1}$ p.s. a los 42 días (Tabla 1). El contenido de nitrógeno total de la retama fresca fue de $42,40 \pm 0,52 \mathrm{~g} \mathrm{~kg}^{-1}$ p.s. y disminuyó de forma ligera y gradual durante el vermicompostaje hasta alcanzar un valor de $36,42 \pm 0,22 \mathrm{~g} \mathrm{~kg}^{-1}$ p.s. a los 42 días (Tabla 1). La relación $\mathrm{C} / \mathrm{N}$ aumentó gradualmente y de forma leve desde un valor inicial de $11,54 \pm 0,17$ hasta un valor final de $12,94 \pm 0,08$ después de 42 días de vermicompostaje (Tabla 1). El contenido en fósforo dela retama fresca fue de 2,93 $\pm 0,32 \mathrm{~g}$ $\mathrm{kg}^{-1}$ p.s. y tras 42 días de vermicompostaje aumentó ligeramente hasta un valor de $3,07 \pm 0,03 \mathrm{~g} \mathrm{~kg}^{-1}$ p.s. El contenido en potasio de la retama fresca fue de 10,33 $\pm 1,19$ $\mathrm{g} \mathrm{kg}^{-1}$ p.s. y disminuyó de forma significativa hasta un valor de $5,99 \pm 0,14 \mathrm{~g} \mathrm{~kg}^{-1}$ p.s. tras 42 días de vermicompostaje (Tabla 1). La retama fresca tenía un contenido en calcio de 
$3,91 \pm 0,4 \mathrm{~g} \mathrm{~kg}^{-1}$ p.s. que aumentó de forma gradual y significativa hasta un valor de $8,99 \pm 0,18 \mathrm{~g} \mathrm{~kg}^{-1}$ p.s. tras los 42 días. El contenido en magnesio de la retama fresca fue de 2,62 $\pm 0,28 \mathrm{~g} \mathrm{~kg}^{-1}$ p.s. y aumentó ligeramente hasta un valor de 2,90 $\pm 0,05 \mathrm{~g} \mathrm{~kg}^{-1}$ p.s. (Tabla 1). El contenido en azufre de la retama fresca fue de $2,38 \pm 0,27 \mathrm{~g} \mathrm{~kg}^{-1}$ p.s. y tras 42 días de vermicompostaje aumentó hasta un valor de $3,20 \pm 0,06 \mathrm{~g} \mathrm{~kg}^{-1}$ p.s. La retama fresca tenía un contenido en hierro de $0,53 \pm 0,07 \mathrm{~g} \mathrm{~kg}^{-1}$ p.s. que aumentó de forma gradual y significativa hasta un valor de 1,89 $\pm 0,02 \mathrm{~g} \mathrm{~kg}^{-1}$ p.s. tras los 42 días (Tabla 1). El contenido en manganeso de la retama fresca fue de $0,35 \pm 0,03 \mathrm{~g} \mathrm{~kg}^{-1}$ p.s. y tras 42 días de vermicompostaje aumentó hasta un valor de $0,64 \pm 0,01 \mathrm{~g} \mathrm{~kg}^{-1}$ p.s. El contenido en boro de la retama fresca fue de $34,2 \pm 3,56 \mathrm{mg} \mathrm{kg}^{-1}$ p.s. y tras 42 días de vermicompostaje aumentó hasta un valor de 40,31 \pm 0,67 $\mathrm{mg} \mathrm{kg}^{-1}$ p.s. La retama fresca tenía un contenido en molibdeno de $1,98 \pm 0,16 \mathrm{mg} \mathrm{kg}^{-1}$ p.s. que aumentó de forma gradual y significativa hasta un valor de 7,65 $\pm 0,26$ $\mathrm{mg} \mathrm{kg}^{-1}$ p.s. tras los 42 días (Tabla 1).

La actividad microbiana de la retama fresca, medida como respiración basal, fue de 779,89 $\pm 9,43 \mathrm{mg} \mathrm{O}_{2} \mathrm{Kg} \mathrm{MO}^{-1} \mathrm{~h}-1$, y disminuyó de forma gradual y significativa hasta un valor de $323,59 \pm 4,14 \mathrm{mg} \mathrm{O}_{2} \mathrm{Kg} \mathrm{MO}^{-1} \mathrm{~h}^{-1}$ tras los 42 días de vermicompostaje (Tabla 1 ).

En lo que respecta a las fibras, el contenido de lignina de la retama fresca fue de $206,04 \pm 4,72 \mathrm{~g} \mathrm{~kg}^{-1}$ p.s y aumentó de forma gradual y significativa hasta un valor de $410,06 \pm 5,86$ $\mathrm{g} \mathrm{kg}^{-1}$ p.s tras los 42 días de vermicompostaje (Tabla 1). La retama fresca tenía un contenido en celulosa de $294,88 \pm 13,89 \mathrm{~g} \mathrm{~kg}^{-1}$ p.s. que disminuyó hasta un valor de $201,54 \pm 5,44 \mathrm{~g} \mathrm{~kg}^{-1}$ p.s. tras los 42 días (Tabla 1). El contenido de hemicelulosa de la retama fresca fue de 258,6 $\pm 0,4 \mathrm{~g} \mathrm{~kg}^{-1}$ p.s y disminuyó de forma gradual y significativa hasta un valor de $84,38 \pm 1,58 \mathrm{~g} \mathrm{~kg}^{-1}$ p.s tras los 42 días de vermicompostaje (Tabla 1 ).

El vermicompostaje es un proceso de biooxidación en el que algunas especies detritívoras de lombrices de tierra interactúan de forma intensa con los microorganismos y otros animales edáficos de la comunidad descomponedora, afectando de forma drástica a los procesos de descomposición, acelerando la estabilización de la materia orgánica y modificando de forma sustancial las propiedades físicas y bioquímicas de la misma (Domínguez 2004; Domínguez et al. 2010). Los microorganismos producen las enzimas responsables de la descomposición bioquímica de la materia orgánica, pero las lombrices son elementos cruciales del proceso y están implicadas en la estimulación indirecta de las poblaciones microbianas a través de la fragmentación y la ingestión de la materia orgánica fresca, lo que aumenta el área superficial disponible para la colonización microbiana, alterando de forma radical la actividad biológica. Las lombrices de tierra también modifican la biomasa y la actividad microbiana a través de la estimulación, digestión y dispersión de los microorganismos e interaccionan con otros componentes biológicos del sistema de vermicompostaje, modificando la estructura de las comunidades de la microbiota y de la microfauna (Domínguez et al. 2010). El vermicompostaje ha resultado ser muy eficaz en el tratamiento de muchos tipos de residuos orgánicos (Domínguez \& Edwards 2011), incluyendo restos vegetales como el bagazo de uva (Martínez-Cordeiro et al. 2013).

En este trabajo, tanto la evolución de la población de lombrices como de los parámetros químicos y biológicos indican que el proceso de vermicompostaje se desarrolló de una forma óptima y rápida, rindiendo un vermicompost de alta calidad. El producto final del proceso, el vermicompost, vermihumus o humus de lombriz es un material similar a la turba, muy particulado y poroso, con alta capacidad de retención de agua, microbiológicamente estabilizado yactivo, con una baja relación $\mathrm{C}: \mathrm{N}$; y constituye una fuente de nutrientes para las plantas, que se liberan poco a poco, a medida que se van mineralizando y la planta los necesita (Domínguez 2004). La cantidad y calidad de los nutrientes en el vermihumus se debe a la mineralización acelerada de la materia orgánica, al aumento de la actividad microbiana, a la degradación y ruptura de los polisacáridos y a las altas tasas de humificación que se alcanzan durante el vermicompostaje (Domínguez \& Gómez-Brandón 2013). Las propiedades biológicas del vermicompost lo conviertenen un excelente fertilizante orgánico; cuando se añade alsuelo o a los medios de cultivo de plantas, el vermihumus aumenta la germinación, el crecimiento, la floración y la producción de fruta y acelera el desarrollo de las plantas. La mejora del crecimiento de las plantas se puede atribuir a varios mecanismos directos e indirectos, que incluyen mecanismos mediados biológicamente, tales como elaporte de sustancias reguladoras del crecimiento de las plantas y la mejora de las funciones biológicas del suelo (Lazcano \& Domínguez 2011).

Evolución del índice de polifenoles totales (IPT) en la retama negra a lo largo del proceso de Vermicompostaje

La cantidad inicial de polifenoles totales fue de $32,44 \pm 1,11$ mg GAE g-1 p.s., y disminuyó rápidamente durante la primera semana, y de hecho un $50 \%$ de los polifenoles desaparecen en ese periodo. A partir del día 7 esta caída es más lenta, aunque progresiva, hasta el día 42 en el que

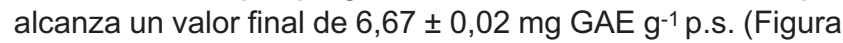
3). La cantidad de polifenoles que queda tras el día 42 es muy baja, de forma que el vermicompost queda libre de polifenoles y está preparado para su uso como biofertilizante. De hecho se considera que uno de los mejores indicadores de la madurez de un vermicompost es la ausencia de polifenoles y de ácidos alifáticos bioinhibidores (Domínguez \& Edwards 2011).

Para comprobar que esta disminución de polifenoles no se debió a su transferencia a la cama del reactor, se analizó también el contenido polifenólico de la misma al inicio y al final del proceso. El valor inicial fue de $3,83 \pm 0,25 \mathrm{mg} \mathrm{GAE}$ $\mathrm{g}^{-1}$ p.s. mientras que el del día 42 fue de $3,15 \pm 0,18 \mathrm{mg}$ GAE g-1 p.s., lo que indica que no hubo pérdidas por transferencia. 


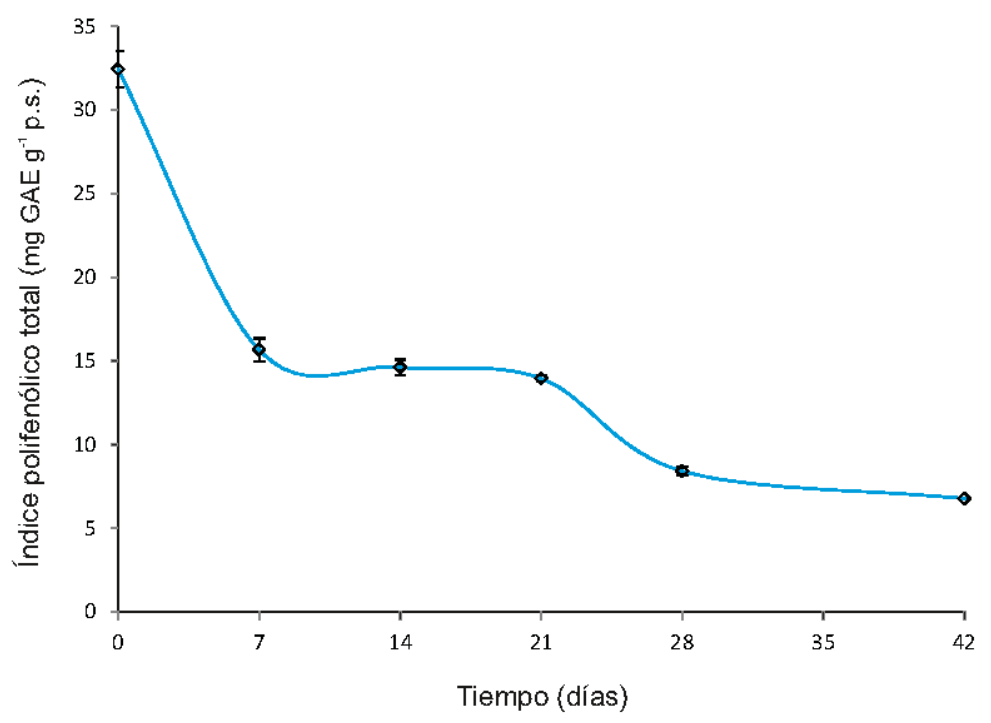

Figura 3.- Evolución del índice de polifenoles totales (IPT) durante el vermicompostaje de retama negra. Los valores son medias \pm error estándar $(n=5)$
Caracterización de los polifenoles individuales en la retama negra y evolución a lo largo del proceso de vermicompostaje

La concentración de los polifenoles individuales disminuyó de forma notable desde el día inicial del proceso hasta el día 42 , si bien esta variación no presentó el mismo perfil para todos los compuestos (Figura 4).

El extracto de la retama negra fresca (día 0) es rico en flavonas, particularmente en crisina y apigenina, que se caracterizan por sus propiedades anti-inflamatorias y antioxidantes. La concentración inicial de apigenina fue de $502 \pm 6,9 \mathrm{mg} \mathrm{kg}^{-1}$ p.s. y disminuyó rápida y progresivamente en las tres primeras semanas, con un valor de $34 \pm 3,3 \mathrm{mg}$ $\mathrm{kg}^{-1}$ p.s. en el día 21 del proceso; quedando el día 42 tan sólo un $1 \%$ de la apigenina inicial (Figura 4a). La apigenina es de enorme interés, por sus propiedades beneficiosas para la salud, por su baja toxicidad y por sus potentes efectos sobre las células cancerosas, en comparación con otros flavonoides estructuralmente relacionados(Shukla \& Gupta 2010). La crisina también es un potente antioxidante, tiene una actividad antimicrobiana elevada y es, además, una flavona con propiedades ansiolíticas demostradas (Gowthamarajan et al. 2002; Liu et al. 2010). La concentración inicial de crisina fue de $1867 \pm 10,2 \mathrm{mg} \mathrm{kg}^{-1}$ p.s., siendo el compuesto polifenólico más abundante en los extractos de retama negra. La evolución de este compuesto se caracteriza, a diferencia de la flavona anterior, por un aumento de la concentración en las dos primeras semanas del proceso de vermicompostaje, alcanzando el doble de la concentración de partida en el día 14 (3732 $\pm 4,5 \mathrm{mg} \mathrm{kg}^{-1}$ p.s.), para disminuir progresivamente a lo largo de las siguientes semanas hasta un valor testimonial del $0,6 \%$ de la crisina original (Figura 4b). Este aumento inicial puede explicarse porque las plantas del género Cytisus contienen varios derivados de la crisina (por ej. O-glucósidos, Cglucósidos, C-hexosilos) (Barros et al. 2012) que se pueden degradar fácilmente en las primeras etapas del vermicompostaje por la labilidad de sus enlaces, liberando crisina como producto de degradación. Este hecho abre otra vía de explotación del proceso de vermicompostaje, ya que la retama recuperada en el día 14 puede utilizarse como una fuente de crisina excepcionalmente rica, con el doble de carga que la materia prima inicial.

El extracto inicial de retama negra se caracteriza también por la presencia de polifenoles no flavonoides, concretamente ácidos fenólicos, aunque en menor concentración que el grupo mayoritario de flavonoides detectados, las flavonas, antes comentadas. La concentración inicial de ácido cafeicofue de $120 \pm 6 \mathrm{mg} \mathrm{kg}$ 1 p.s. y disminuyó muy rápidamente, a un tercio de la cantidad inicial en los primeros 7 días (41 $\pm 3 \mathrm{mg} \mathrm{kg}^{-1}$ p.s.) y hasta un valor final de $9 \pm 3 \mathrm{mg} \mathrm{kg}^{-1}$ p.s. en día 42 (Figura 4c). La concentración inicial de ácido protocatéquico fue de $56 \pm 6 \mathrm{mg} \mathrm{kg}^{-1}$ p.s., pero su perfil de biodegradación fue más lento, detectándose $23 \pm 2 \mathrm{mg} \mathrm{kg}^{-1}$ p.s. en los extractos del día 42, aproximadamente la mitad de la concentración de partida(Figura 4d). Los perfiles de biodegradaciónde cada polifenol son específicos y dependen de su biodisponibilidad y facilidad para ser digeridos;si bien existe una clara tendencia general de disminución, más acusada durante los primeros días del proceso. Estos resultados concuerdan con los observados para el índice de polifenoles totales, donde los valores también disminuian de forma más rápida la primera semana.

\section{Conclusión}

El vermicompostaje ha demostrado ser un proceso muy rápido y eficaz para la transformación de la retama negra en un biofertilizante de alta calidad. En tan solo 42 días se obtiene un vermicompost, vermihumus o humus de lombriz, que es un material similar a la turba, muy particulado y poroso, con alta capacidad de retención de agua, microbiológicamente estabilizado yactivo, con una baja relación $\mathrm{C}: \mathrm{N}$; y que constituye una fuente de liberación lenta 